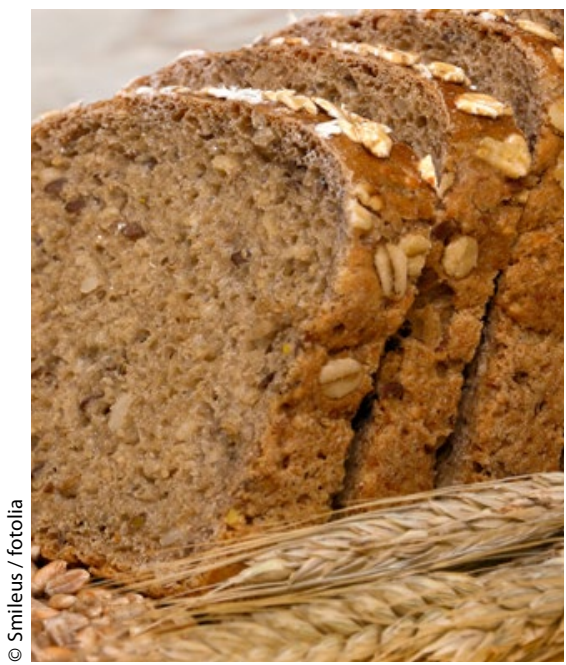

Wer Vollkornbrot isst, lebt länger - so das Fazit dieser Studie. oder nur teilweise im Darm abgebaut werden können. $100 \mathrm{~g}$ Vollkornbrot enthalten etwa $8 \mathrm{~g}$ Ballaststoffe. Unumstritten ist, dass Ballaststoffe Stoffwechselkrankheiten und kardiovaskulären Krankheiten sowie Krebs und Diabetes vorbeugen und die Mortalität senken. Die Deutsche Gesellschaft für Ernährung empfiehlt einen Konsum von über $30 \mathrm{~g} / \mathrm{Tag}$.

Wie stellt man sich die zugrunde liegenden Mechanismen vor? Ballaststoffe generieren kaum oder keine Kalorien, sättigen und beugen daher Übergewicht und Adipositas vor. Dem Darm entziehen Ballaststoffe geschädigte Zellen, was das Risiko für Kolonkarzinome vermindert. Vollkornmehl vermindert im Vergleich zu Weißmehl postprandiale Glukose- und Insulinspiegel, senkt den Blutdruck, Triglyzeride sowie
Gesamt- und LDL-Cholesterin und inflammatorische Marker (z. B. CRP).

Fazit: Diese sowie vorausgehende Metaanalysen belegen, dass Vollkorn jedermann zu empfehlen ist. In Schweden wird ein Konsum von mindestens $75 \mathrm{~g} / \mathrm{Tag}$ (Trockengewicht) empfohlen.



Prof. Dr. med. Alfred Wirth

Bad Rothenfelde

\section{Wie sicher sind NOAK bei älteren Patienten mit Niereninsuffizienz?}

\begin{abstract}
Patienten jenseits des 80. Lebensjahres, die an Niereninsuffizienz oder Untergewicht leiden, erhalten bei Vorhofflimmern häufig niedrige Dosen eines neuen oralen Antikoagulans, obwohl sich diese Empfehlung nur auf wenige klinische Daten stützt.
\end{abstract}

\begin{abstract}
$A$ us dem landesweiten dänischen Register wurden die Daten aller Patienten entnommen, die wegen Vorhofflimmern zur Schlaganfallprävention erstmals mit einem der neuen oralen Antikoagulanzien (NOAK) in niedriger Dosierung oder wie üblich mit Warfarin ( $\mathrm{n}=38.893)$ behandelt wurden. Eine Dosisreduktion (Apixaban: 2 $\times 2,5 \mathrm{mg}, \mathrm{n}=4.400$; Dabigatran: $2 \times$ $110 \mathrm{mg}, \mathrm{n}=8.875$ oder Rivaroxaban: 1 $\times 15 \mathrm{mg}, \mathrm{n}=3.476)$ erfolgte entsprechend den Empfehlungen wegen hohen Alters über 80 Jahren, eingeschränkter Nierenfunktion (GFR zwischen 15-30
\end{abstract}

und $50 \mathrm{ml} / \mathrm{min}$ ) oder Gewicht unter 60 $\mathrm{kg}$.

Ischämische Schlaganfälle und periphere Embolien waren nach einjähriger Therapie unter der reduzierten Dosis von $2 \times 2,5 \mathrm{mg}$ Apixaban täglich mit $4,8 \%$ zwar häufiger als unter Warfarin mit 3,7\%, $2 \times 110$ mg Dabigatran mit 3,3\% und $1 \times 15 \mathrm{mg}$ Rivaroxaban mit 3,5\%, aber in keinem Fall signifikant unterschiedlich (Tab. 1).

Im Vergleich zu Warfarin waren Blutungen unter Dabigatran signifikant seltener, während es zu Apixaban und Rivaroxaban keinen Unterschied gab.

\begin{tabular}{|c|c|c|c|c|}
\hline & Apixaban & Dabigatran & Rivaroxaban & Warfarin \\
\hline Thromboembolien & $4,8 \%$ & $3,3 \%$ & $3,5 \%$ & $3,7 \%$ \\
\hline Blutungen & $5,1 \%$ & $4,1 \%$ & $5,6 \%$ & $5,1 \%$ \\
\hline
\end{tabular}

